\title{
Energy Efficient Multiplier Design Using Gdi Logic
}

\author{
K.Sai srilakshmi ${ }^{1}$ V.Nancharaiah ${ }^{2}$ Ch.Karthik ${ }^{3}$ K.Jagadeesh ${ }^{4}$ \\ ${ }^{13}{ }^{4}$ Student, Department of ECE, Lendi Institute of Engineering and Technology \\ ${ }^{2}$ Associate professor, Department of ECE,Lendi Institute of Engineering and Technology
}

\begin{abstract}
Addition and multiplications are a fundamental blocks to perform all operations in digital circuits. A high performance multiplier is a one of the key component in designing applications of integrated circuits. In designing integrated circuits power is a one of premium resources a designer tries to save when designing the circuit or system.The major issue for design of adderpower dissipation, delay. These issues can be overcome by new technique GATE DIFFUSION INPUT (GDI) logic technique which is commonly occurring a threshold voltage problem, so it does not allow adders without additional inverters. In this paper three full adders designed with full swing gates by using GDI logic called as proposed designs. The proposed designs are improve in their circuit performance. Performance of proposed designs having a low power consumption when compared all other designs in simulation results. The goal is to proposed full adders extended with multiplier to reduce the complexity of design as well as make a low energy consumption and high speed in designing systems.

Keywords: Adder, GDI logic,Digital design, Full swing.
\end{abstract}

\section{Introduction}

Due to rapid development of portable applications, electronics market is becoming more competitive, which results in consumer electronic products requiring even more stringently high quality. Therefore, the integrated circuit (IC) designers have to consider more important issues such as chip area, power consumption, operation speed, circuit regularity, and so on [15].In most of the digital circuits, adders performs a major role which influences the overall system performance. Addition is a fundamental block to perform any operation that is broadly used in many VLSI systems, such as application-specific digital signal processing (DSP) and microprocessors architectures[4].Power consumption and speed would be the othertwo important criteria when it comes to the design of full adders. The design of a full-adder having low-power consumption and low propagation delay results of great interest for the implementation of modern digital systems. Therefore, power delay product or energy consumption per operation has been introduced to accomplish optimal design trade-offs. The performance of digital circuits can be optimized by proper selection of logic styles.In this paper we are designed low power consumption adders extended with multiplier to reduce the complexity and reduce the power consumption when compared with other designs. This may reduce overall capacitances which in turn will increase the speed and decrease the power dissipation. The adder design implemented using transmission gate with a new technique GDI (Gate Diffusion Input) . This technique allows reducing power consumption, delay and area of a low power digital circuits, while maintaining low complexity of logic design[12].

\section{Gdi Logic}

Gate diffusion input (GDI) - a new technique of low-power digital combinatorial circuit design is described. This technique allows reducing power consumption, propagation delay, and area of digital circuits while maintaining low complexity of logic design. Performance comparison with traditional CMOS and various pass-transistor logic design techniques is presented. The different methods are compared with respect to the layout area, number of devices, delay, and power dissipation. Issues like technology compatibility, top-down design, and precomputing synthesis are discussed, showing advantages and drawbacks of GDI compared to other methods. Several logic circuits have been implemented in various design styles. Their properties are discussed, simulation results are reported, and measurements of a test chip are presented.Basic GDI logic input as shown in figure.

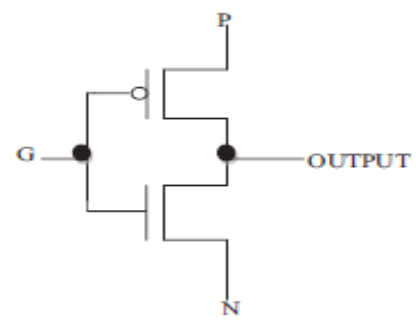

FIGURE 1:-GDI CELL 
At first glance, the basic cell reminds one of the standard CMOS inverter, but there are some important differences.1) The GDI cell contains three inputs:G(common gate input of nMOS and pMOS), $\mathrm{P}$ (input to the source/drain of pMOS), and $\mathrm{N}$ (input to the source/drain of nMOS). 2) Bulks of both nMOS and pMOS are connected to $\mathrm{N}$ or $\mathrm{P}$ (respectively), so it can be arbitrarily biased at contrast with a CMOS inverter.

Most of these functions are complex (6-12 transistors) in CMOS, as well as in standard PTL implementations, but very simple (only two transistors per function) in the GDI design method. In this paper, most of the designed circuits were based on the F1 and F2 functions. The reasons for this are as follows.

1) Both F1 and F2 are complete logic families (allows realization of any possible two-input logic function).

2) F1 is the only GDI function that can be realized in a standard p-well CMOS process, because the bulk of any nMOS is constantly and equally biased.

3) When $\mathrm{N}$ input is driven at high logic level and $\mathrm{P}$ input is at low logic level, the diodes between NMOS and PMOSbulks to Out are directly polarized and there is a shortbetween $\mathrm{N}$ and $\mathrm{P}$, resulting in static power dissipation and $\mathrm{V}_{\mathrm{OUT} \sim} 0.5 \mathrm{~V}_{\mathrm{DD}}$.

\section{PROPOSED ADDER IN GDI:-}

Three proposed full adder designs featured with GDI full swing gates with the goal to minimize the circuit complexity and to achieve speed at cascaded operation. The strategy is to avoid threshold voltage losses with the help of full swing gatesswing gates.

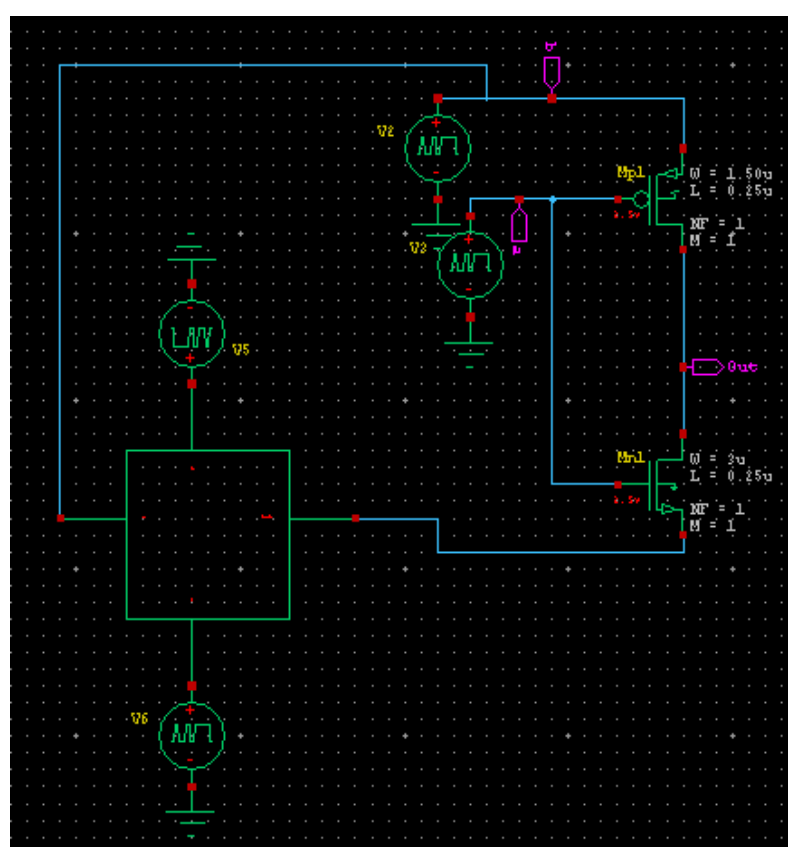

FIGURE 2:-Xor gate
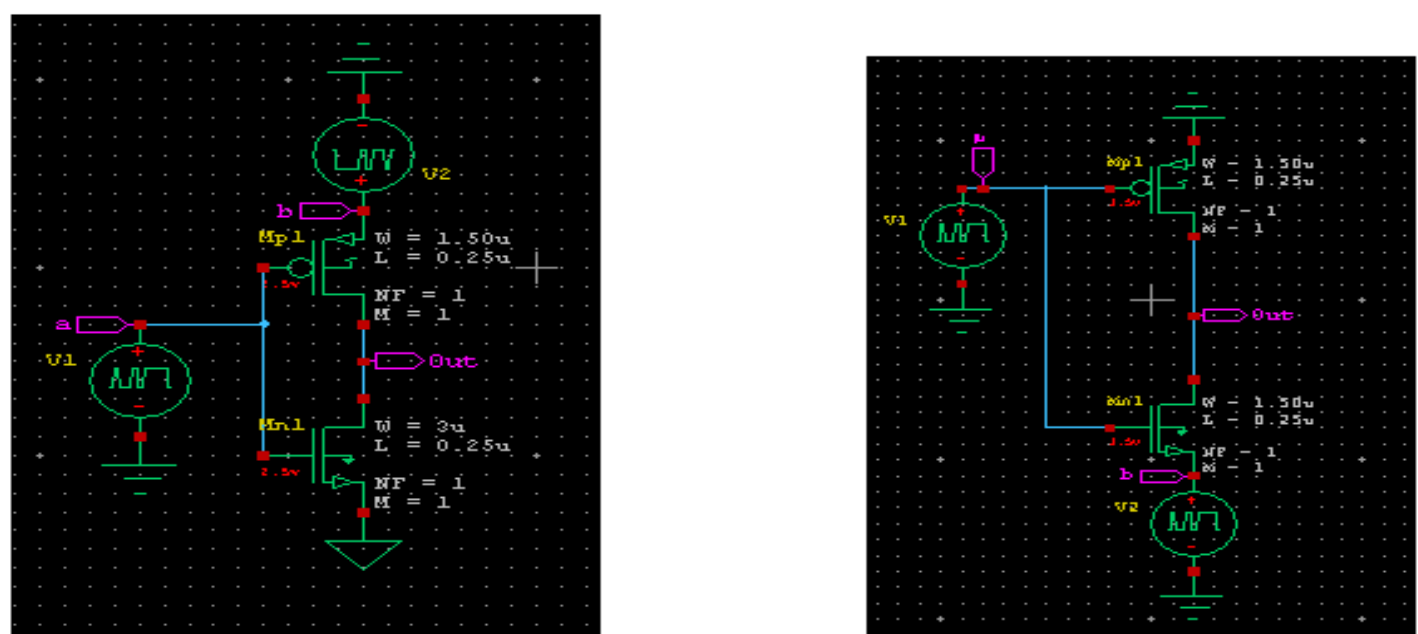

FIGURE 3:-Or gate 


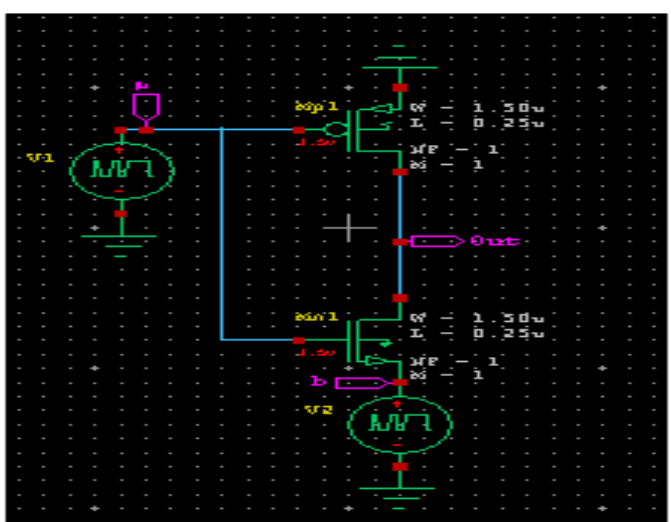

FIGURE 4:-And gate

The logic function of full adder can be represented as below equations

Sum $=$ A XOR B XOR Cin Cout $=$ A AND B + B AND Cin + A AND Cin

From above equations three basic gates are needed for implementing the function i.e., AND, OR and XOR with their transistor level diagrams. The operational characteristics of these gates are given table. The output voltages are degraded by threshold voltage drop for certain input combinations. The reduction in output voltage increases significantly withincrease in the number of stages.

\section{FULL ADDER DESIGNS:-}

The full adders are design of GDI logic with the help of full swing gates using AND, OR, XOR. This design completely eliminates the full swing restoration buffers that results in improve in their performance. Three designs are rewrite by the full adder expressions as listed below

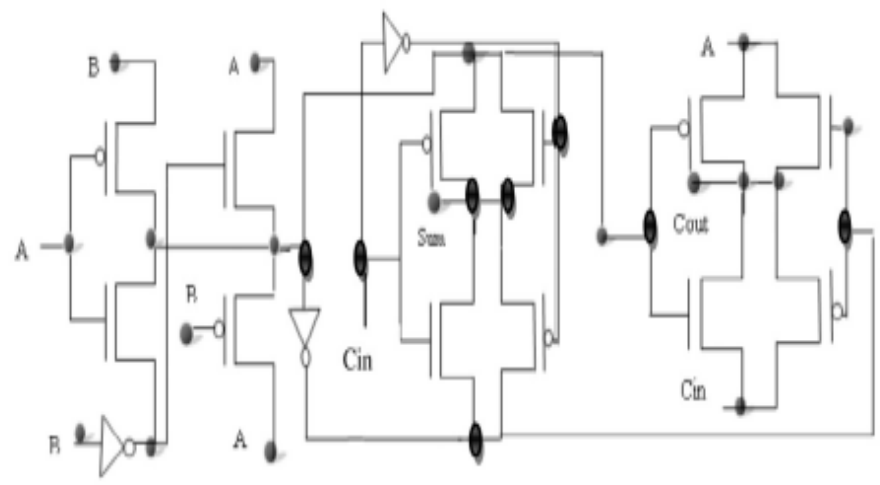

FIGURE 5:-Design1

Design1 Full adder having a output expressions for Sum and Cout with respective below equations 3,4

$\operatorname{Sum}=\operatorname{Cin}(A$ XOR B $)+\operatorname{Cin}(A$ XNORB $)$ Cout $=($ A XOR B $)$ Cin $+($ A XOR B $)$ A

Design 1 uses XOR output as an intermediate result for computing Sum and Cout. Sum output can be attained by multiplexing the XOR and its inverted version XNOR through Cininput. The Coutis obtained by multiplexing the inputs $A$ and $C$ inwhose output is controlled by the selection input, i.e. XOR output of $A$ and $B$ inputs. The presence of inverter on the critical path increases the delay of the whole circuit. This design is simple and requires a total of 18transistors for realizing the full adder function.

Design 2 Full adder having a output expressions for sum and cout but there is a difference in design. This design of full adder consists of XOR, AND, OR Gates when compared to previous design.

$\operatorname{Sum}=$ A XOR B XOR Cin(5)

Cout $=\operatorname{Cin}(A$ AND B $)+\operatorname{Cin}(A$ OR B $)(6)$ 


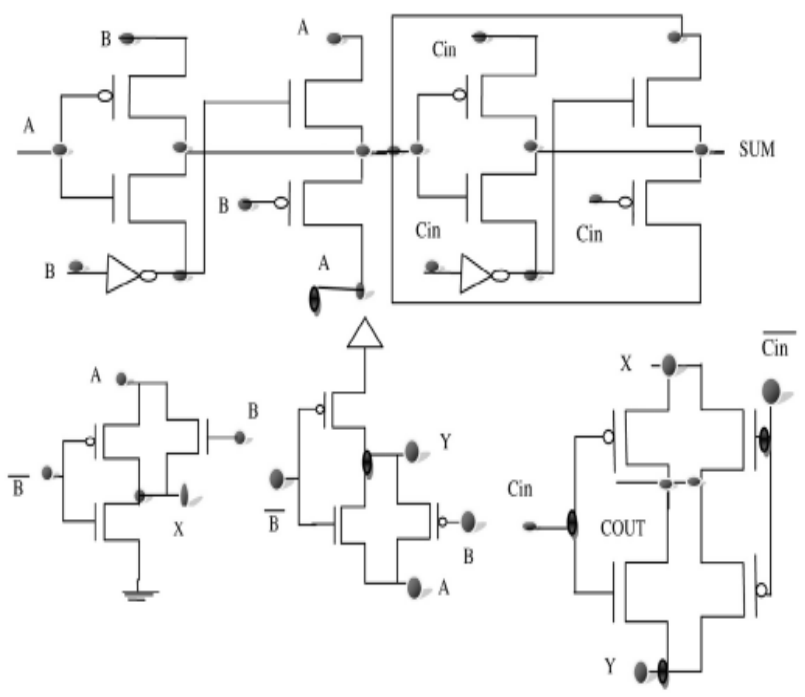

FIGURE 6:-Design 2

Design 2 based full adder. AND and OR gates are designed based on F1 and F2, respectively. Multiplexing the AND andOR operation through Carry input Cinhelps in Coutrealization.The XOR operation on the inputs $A, B$ and Cinachieves Sum function.

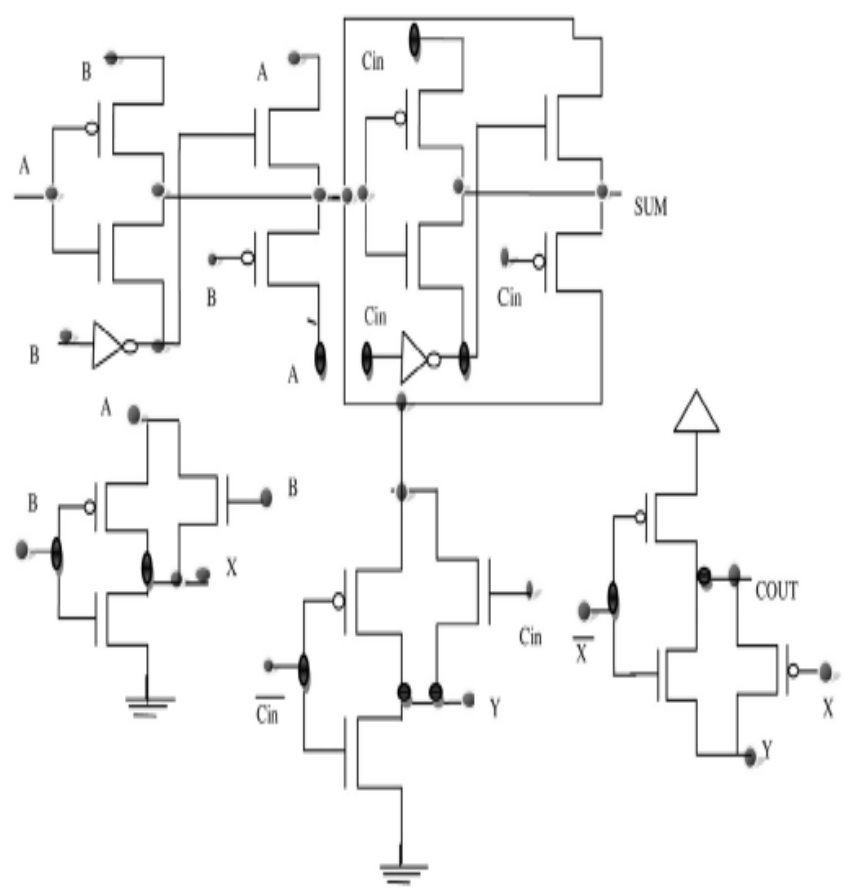

FIGURE 7:-Design 3

Design 3 Full adder having a output expressions for sum and cout with respective equations 7, 8

Sum $=$ A XOR B XOR Cin(7)

Cout $=$ A AND B + (A XOR B $)$ Cin $(8)$

Design 3uses XOR module that plays an important role since Sum output can be achieved by XORing the inputs $A, B$ and $C i n$. The outputCoutis obtained with the help of AND and OR followed by XOR gate.The realization of AND and OR gate can be done with the help offull swing F1 and F2 gates. The GDI based F1 and F2 enables the implementation of AND and OR with only 3 transistors where as CMOS needs 6 transistors for achieving the same. The intermediate XOR gate output is used for computing Coutoutput.

CMOS FULL ADDER DESIGN:- 


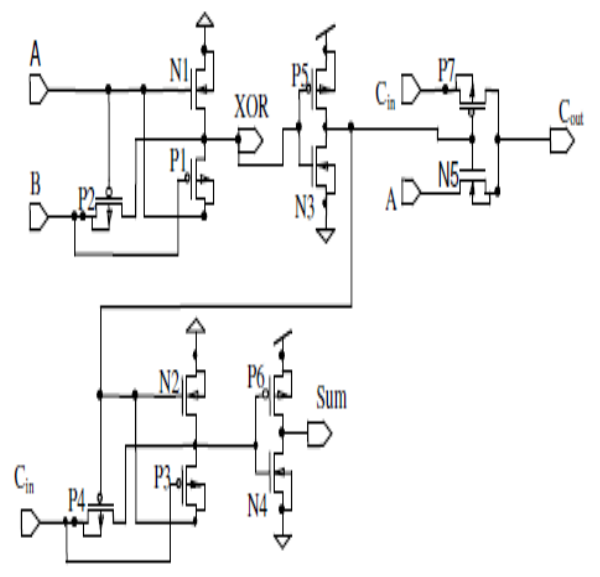

FIGURE 8:- Design4

In this design Cout (carry out) signal has been generated by two transistor multiplexer block with Cin, A and XNOR signal. Sum signal is generated with XNOR signal generated by inverter and Cin signal. This circuit contains 12 transistors with xor gates.As CMOSinverter is responsible for major portion of power consumption in this adder circuit.

These designs are extended with multipliers for low energy consumption.

\section{MULTIPLIERS:-}

Multipliers play an important role in today's digital signal processing and various other applications. With advances in technology, many researchers have tried and are trying to design multipliers which offer either of the following design targets - high speed, low power consumption, regularity of layout and hence less area or even combination of them in one multiplier thus making them suitable for various high speed, low power and compact VLSI implementation.

The common multiplication method is "add and shift" algorithm. In parallel multipliers number of partial products to be added is the main parameter that determines the performance of the multiplier. To reduce the number of partial products to be added, Modified Booth algorithm is one of the most popular algorithms. To achieve speed improvements Wallace Tree algorithm can be used to reduce the number of sequential adding stages. Further by combining both Modified Booth algorithm and Wallace Tree technique we can see advantage of both algorithms in one multiplier. However with increasing parallelism, the amount of shifts between the partial products and intermediate sums to be added will increase which may result in reduced speed, increase in silicon area due to irregularity of structure and also increased power consumption due to increase in interconnect resulting from complex routing. On the other hand "serial-parallel" multipliers compromise speed to achieve better performance for area and power consumption. The selection of a parallel or serial multiplier actually depends on the nature of application. In this lecture we introduce the multiplication algorithms and architecture and compare them in terms of speed, area, power and combination of these metrics. Based on full adder designs $3 * 3$ multipliers are designed.

\section{3*3MULTIPLIER:-}

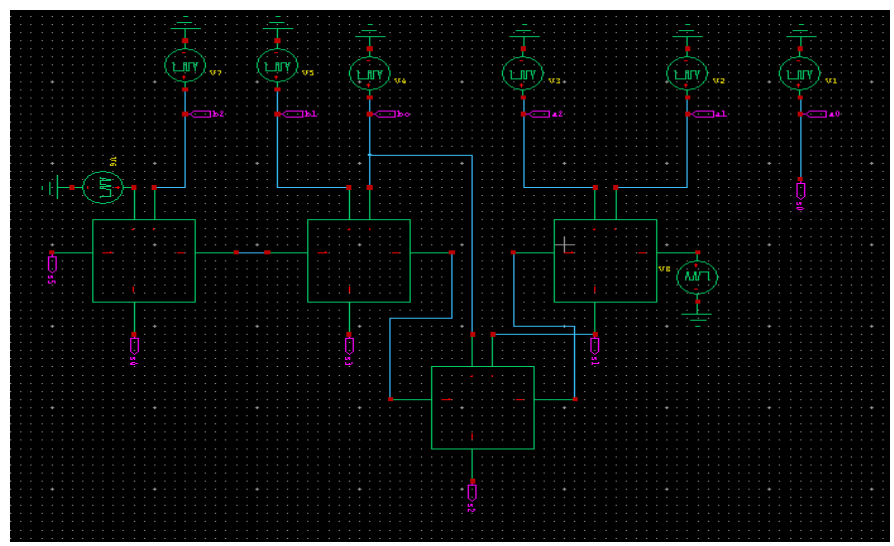

FIGURE 9:- 3*3 Multiplier 


\section{SIMULATION RESULTS FOR MULTIPLIERS:-}

For simulation environment Full adders based on other logic are taken for comparison with the proposed designs.In addition to these adders based on XOR gate are also taken into account. It has a full voltage swing and buffered Sum and Coutsignals. It uses the feedback transistors for providing full swing. The design which uses the combination of CMOS and PTL to generate Sum and Cout,respectively is called hybrid design. It uses 24 transistors in this regard, which lies between CMOS and transmission gate. It is very clear that CPL logic consumes relatively more power due to more number of transistors required for its design. In the case of hybrid design, this equally performs well with CMOS in terms of delay and power consumption. However, it takes reduced number of transistor count compared to CMOS for its design. Whereas the Three proposed GDI based full adders, especially Design 2 outperforms all the other adders in both delay and Energy Delay Product (EDP). This would have resulted due to reduced transistor count on the paths between input and output. This will also lead to the decrease in the parasitic capacitance at the Sum and Coutnodes.The performance metrics of all the simulated adders such as delay, power consumption, energy consumption and process variation analysis are discussed.Three proposed full adder designs have advantages and also limitations. Design 1 is an optimal candidate for the applications in which minimum transistor count and low power is a design requirement. The Design 2 provides lower EDP and minimum delay, so it can be suitable for battery operated and real-time applications. It has slightly increased in transistor count compared with Design 1. Design 3 lies midway between Design 1 and Design 2, and offers lowerdelay than Design 1 as shown in results from given table

\section{TABLE 1}

\begin{tabular}{|l|l|l|l|}
\hline Logic & $\begin{array}{l}\text { Power } \\
\text { (uw) }\end{array}$ & $\begin{array}{l}\text { Delay } \\
\text { (ns) }\end{array}$ & Transistors \\
\hline Design1 & 3.26 & 15.49 & 18 \\
\hline Design2 & 107.36 & 0.164 & 22 \\
\hline Design3 & 32.49 & 19.86 & 23 \\
\hline
\end{tabular}

From the obtained results, it can be concludedthat all three designs operate with less energy consumptionthan existing adders taken for comparison. Hence these design can be extended with multiplier to reduce the complexity to make an low energy consumption circuits in system design. Multiplier power analysis as shown in table.

TABLE 2

\begin{tabular}{|l|l|l|l|}
\hline Design & $\begin{array}{l}\text { Power } \\
\text { (uw) }\end{array}$ & $\begin{array}{l}\text { Delay } \\
\text { (ns) }\end{array}$ & PDP \\
\hline $\begin{array}{l}3 * 3 \text { Multiplier } \\
\text { using Design1 }\end{array}$ & 146.43 & 0.313 & 0.045 \\
\hline $\begin{array}{l}3 * 3 \text { Multiplier } \\
\text { using Design2 }\end{array}$ & 4114.66 & 19.97 & 82.16 \\
\hline $\begin{array}{l}3 * 3 \text { Multiplier } \\
\text { using Design3 }\end{array}$ & 451.27 & 19.73 & 8.903 \\
\hline $\begin{array}{l}3 * 3 \text { Multiplier } \\
\text { using cmos }\end{array}$ & 0.130 & 19.90 & 2.587 \\
\hline
\end{tabular}

\section{Conclusion}

In this paper $3 * 3$ multiplier is proposed based on GDI full adder designs. Out of the three designs based multipliers design1 is superior than other two designs. Comparing with cmos based multiplier and design2, design3 based multipliers, design1 based multiplier has less delay. The power delay product of design1 is 0.045 . 


\section{References}

[1]. Mohan Shoba, RangaswamyNakkeeran GDI based full adders for energy efficient arithmetic applications,

[2]. A. Chandrakasan, R.W. Broderson, Low Power Digital CMOS Design, Kluwer Academic Publishers, 2002.

[3]. V.G. Oklobdzija, D. Villeger, Improving multiplier design using improved columncompression tree and optimized final adder inCMOS technology, IEEE Trans. VLSI Syst. 3 (2) (1995) 292-301.

[4]. A.M. Shams, D.K. Darwish, M.A. Bayoumi, Performance analysis of low power 1-bit CMOS full adder cells, IEEE Trans. VLSI Syst. 10 (1) (2002) 20-29.

[5]. M. Maeen, V. Foroutan, K. Navi, On the design of low power 1 -bit full adder cell, IEICE Electron. Expr. 6 (16) (2009) 11481154.

[6]. S. Purohit, M. Margala, Investigating the impact of logic and circuit implementation for fulladder performance, IEEE Trans. VLSI Syst. 20 (7) (2012)1327-1331.

[7]. P.M. Lee, C.H. Hsu, Y.H. Hung, Novel 10-T full adders realized by GDI structure,Proc. IEEE Int. Symp. Integrated. Circuits (2007) $115-118$.

[8]. I. Hassoune, D. Flandre, I. O’Connor, J. Legat, ULPFA: a new efficient design ofa power-aware full adder, IEEE Trans. Circuits Syst. 57 (8) (2010) 2066-2074.

[9]. M. Aguirre-Hernandez, M. Linares-Aranda, CMOS full adders for energy efficientarithmeticapplications, IEEE Trans. VLSI Syst. 19 (4) (2011) 718-721.

[10]. [10] A. Morgenshtein, A. Fish, I.A. Wagner, Gate Diffusion Input (GDI)-A powerefficient method for digital combinatorial circuits, IEEE Trans. VLSI Syst. 10 (5)(200

[11]. 2) 566-581.

[12]. R. Uma, P. Dhavachelvan, Modified gate diffusion input technique: a newtechnique for enhancing performance in full adder circuits, Proc. ICCCS (2012)74-81.

[13]. A. Morgenshtein, I. Shwartz, A. Fish, Full swing Gate Diffusion Input (GDI) logic- case study for low power CLA adder design, Integration (Amst) 47 (1) (2014)62-70.

[14]. S.Wariya, R. Nagaria, S. Tiwari, Performance analysis of high speed hybrid CMOSfull adder circuits for low voltage VLSI design, VLSI Design (2012) 1-18

[15]. Chiou-Kou Tung, Shao-Hui Shieh2 and Ching-HwaCheng :A Regularly Modularized Multiplexer-based Full Adder for Arithmetic Applications(2014). 